\title{
GESTÃO DE UNIVERSIDADES E TECNOLOGIAS CAFM ${ }^{1}$
}

\author{
Roberta Pinto Teles \\ Universidade Federal da Bahia (UFBA) \\ arq.robertateles@gmail.com
}

UNIVERSITIES MANAGEMENT AND CAFM TECHNOLOGY

\begin{abstract}
Resumo
Este artigo visa fomentar a discussão sobre a tecnologia Computer-aided Facility Management (CAFM) na operação e manutenção de universidades. O avanço tecnológico associado ao incremento de recursos e equipamentos nas edificações, aliado aos novos valores da sociedade para uma série de exigências, estabeleceu uma rotina que requer condições de uso fundamentado na operação e manutenção mais eficientes e eficazes, buscando o funcionamento num elevado nível de serviço. A introdução e o uso do paradigma BIM, implica num processo que propõe significativas mudanças na área da construção civil, onde se destaca o elemento "Informação" durante o ciclo de vida da edificação, desde a fase inicial do empreendimento e execução da obra, evidenciando-se a importância da etapa de operação e manutenção, dada a sua duração e custos envolvidos. Este estudo descreve alguns modelos de gestão de universidades que utilizam a tecnologia CAFM e relata o início do estudo de caso da pesquisa em andamento sobre a aplicação de CAFM na Faculdade de Arquitetura - FAUFBA. A implantação de CAFM envolve a participação de diversos setores para elicitar o atual modelo de gestão e possibilitar a formulação de uma nova proposta de gestão, evitando gastos desnecessários e descontinuidade de serviços de manutenção.
\end{abstract}

Palavras-chave: Gestão de Facilidades. CAFM. Universidade.

\begin{abstract}
This article is intended to foment discussion of the Computer-aided Facility Management Technology (CAFM) in the operation and maintenance of universities. Technological advances associated with increased resources and equipment in buildings, together with the new values of society for a number of requirements, established a routine that requires substantiated conditions of use in the most efficient and effective operation and maintenance, operation seeking a high level of service. The introduction and use of the BIM paradigm implies a process that proposes significant changes in the construction area, which highlights the element "information" during the life cycle of the building, from the initial phase of the development and execution of the work, showing If the importance of the operation and maintenance stage, given the duration and the costs involved. This study describes some models of management of universities that use the CAFM technology and reports the start of the research case study in progress on the implementation of CAFM in the School of Architecture - FAUFBA. The implementation of CAFM involves the participation of various sectors to elicit the current management model and enable the formulation of a new proposal management, avoiding unnecessary expenses and discontinuation of maintenance services.
\end{abstract}

Keywords: Facilities Management. CAFM. University.

\footnotetext{
1 TELES, R. P. Gestão de universidades e tecnologias CAFM. In: ENCONTRO BRASILEIRO DE TECNOLOGIA DE INFORMAÇÃO E COMUNICAÇÃO NA CONSTRUÇÃO, 7., 2015, Recife. Anais... Porto Alegre: ANTAC, 2015.
} 


\section{INTRODUÇÃO}

Este texto discute o uso da tecnologia Computer-aided Facility Management (CAFM) na gestão de universidades, na sua maioria estrangeiras, onde o emprego desta tecnologia é bastante difundido. Pode-se destacar neste processo uma rotina de participação da comunidade acadêmica em algumas decisões relevantes no processo de gestão.

Para entender melhor o espaço das universidades, é importante estudar a sua origem nos centros das cidades europeias, até o surgimento do padrão americano de instalações no campo, distantes dos centros urbanos, onde surge o termo campus universitário. Em seguida, o Brasil com o campus universitário adaptado às condições locais. Neste caso, a designação campus ou cidade universitária caracterizam o mesmo espaço, que geralmente não funcionam de forma independente dos centros urbanos, eles costumam se apoiar na estrutura das cidades de uma forma geral nos serviços de transporte, moradia, supermercados, farmácias, que dão apoio aos estudantes, funcionários e professores dos centros de ensino superior.

As contínuas transformações organizacionais sob o aspecto cultural e social refletem diretamente no uso e ocupação do espaço. Como consequência, a cada dia a tecnologia associada às novas edificações gera uma série de desafios no âmbito da operação e manutenção, envolvendo equipamentos de última geração, sistemas de automação, segurança contra incêndio, segurança patrimonial, refrigeração, circulação vertical etc. Este grau de complexidade é agravado em decorrência da área extensa, composto por edificações com variadas funções, áreas verdes, estacionamentos etc.

Segundo Antonioli (2003), novas e complexas solicitações são impostas a cada momento. Critérios de desempenho e qualidade passam a integrar o universo de exigências impostas ao edifício e a seus sistemas, obrigando a operação destes em patamares cada vez mais elevados de confiabilidade e flexibilidade.

Este trabalho faz parte de uma pesquisa de mestrado em fase de desenvolvimento, onde está sendo elaborado um modelo BIM da Faculdade de Arquitetura da UFBA para aplicações em Facilities Management. Com isto pretende-se propor uma rotina de procedimentos desta aplicação, buscando aumentar a eficiência da edificação, reduzir custos operacionais, facilitar a tomada de decisões administrativas e prolongar a vida útil do empreendimento e seus equipamentos. Nesta primeira etapa estamos elaborando o levantamento das atividades que poderão ser atendidas pelo sistema CAFM, as pessoas e os ambientes relacionados a estas atividades. Inicialmente pretende-se aplicar estes recursos no controle de alocação de espaços de sala de aula, para isso, estamos definindo o código de identificação de cada espaço.

Na perspectiva atual de gestão do território, planejamento, ordenação e monitoramento do espaço deve incluir a análise dos diferentes componentes do ambiente, incluindo o meio físico-biótico, a ocupação humana, e seu inter-relacionamento. Tais recursos podem ser utilizados para caracterizar e entender a organização do espaço, como base para o estabelecimento de ações e estudos futuros (CÂMARA; MEDEIROS, 1998).

A implantação da tecnologia CAFM envolve a participação de todos os setores da organização visando contribuir para a elicitação do atual modelo de gestão e possibilitar a formação de um banco de dados com informações úteis, visando a formulação de uma proposta de gestão, mais adequada às necessidades acadêmicas.

\section{GESTÃO DE FACILIDADES}

Segundo Quinello e Nicoletti (2006) a palavra facilidade vem do latim facilitas - atis referindo-se ao ato de auxiliar e tornar mais fácil alguma ação. Já a "gestão de facilidades" 
seria a combinação de esforços para facilitar as atividades de uma organização. O ideal da gestão de facilidades é a constante busca por redução de custos operacionais e de manutenção buscando a eficiência dos serviços e diminuição de riscos e fracassos.

Conforme Quinello e Nicoletti (2006), a estruturação do projeto de gestão depende de vários fatores: complexidade, porte, disponibilidade de pessoas e de outros recursos. Geralmente o líder do projeto define a gestão mais adequada, se descentralizada, agrupada ou centralizada pelo seu departamento. O importante é que exista uma convergência de objetivos, para o projeto ser executado de forma harmônica.

\section{TECNOLOGIA CAFM (COMPUTER-AIDED FACILITY MANAGEMENT)}

Pela definição do International Facilities Management Association (IFMA) ${ }^{2}$ (2015), gestão de "facilidades" é uma atividade que engloba múltiplas disciplinas, para assegurar a funcionalidade do ambiente construído, por meio da integração de pessoas, locais, processos e tecnologia.

Segundo Watson (2013) esta tecnologia é a combinação de Computer-Aided Design (CAD) e recursos de banco de dados com habilidades específicas para Facilities Management (FM). A finalidade deste sistema inclui: ajudar o gerente da instalação a garantir que os ativos de uma organização sejam utilizados com o menor custo possível, ao fornecer benefícios para todas as fases do ciclo de vida de um edifício e apoiar a gestão da instalação operacional e estratégica.

Watson (2013) afirma que os sistemas CAFM geralmente são compostos por uma variedade de tecnologias e fontes de informações que podem incluir sistemas orientados a objetos de banco de dados, sistemas de CAD, Building Information Modeling (BIM), e das interfaces com os outros sistemas, como o Sistema Informatizado de Gestão de Manutenção (CMMS).

Ferreira (2005) diz que ainda não foi encontrado um termo em português para traduzir adequadamente os conceitos de facilities, que pode englobar a gestão da edificação, de pessoas, de espaço, de processos de trabalho e dos ativos de uma organização.

O Facility Management tem como objetivo a melhoria da utilização dos recursos de uma instalação facilitando o seu desempenho. Esta pode ser desenvolvida a partir de sistemas automatizados de coleta e controle de dados, e também por meio de conceitos e procedimentos rotineiros de gestão, dentre outras disciplinas. Com os sistemas CAFM é possível monitorar as instalações com informações detalhadas dos componentes e sistemas, intervalos de manutenção, custos e reparos dos equipamentos (WEISE, 2009).

Computer-aided Facility Management - Uso de metodologias assistidas por computador, incluindo desenhos, ferramentas de relatórios de bancos de dados e planilhas para gerenciar grandes quantidades de informação relacionada com as facilities (IFMA, 2013, p. 1, tradução nossa)

Esta tecnologia pode atender a clientes diversificados, desde órgãos do governo, da área de saúde, educação, comércio e ambientes industriais. Os estabelecimentos universitários oferecem aos gestores uma série de desafios por serem instalações geralmente amplas, com diversidade de edificações e diferentes funções, tais como: laboratórios de diferentes tipos, bibliotecas, salas de aula, auditórios, refeitórios, residências universitárias, administração, esporte e lazer.

Com a modelagem de dados e o seu carregamento é possível disponibilizar recursos para auxiliar o desempenho da atividade de gestão de facilities, tais como:

\footnotetext{
2 IFMA - International Facilities Management Association, Associação Internacional para os Profissionais de Gestão de Instalações (tradução nossa).
} 
- Gerenciamento do espaço, ocupação, alterações de layout, manutenção, locação de imóveis;

- Controle de custos e de atividades de manutenção;

- Satisfação dos usuários;

- Sistema Help Desk ${ }^{3}$ - serviço de apoio a usuários para suporte e resolução de problemas técnicos;

- Controle de ativos - um ativo é um item, coisa ou entidade que tem valor real ou potencial para uma organização, sendo essencial manter o controle do mesmo ao longo do seu ciclo de vida, ou seja, da aquisição até o seu descarte (ANTONIOLI, 2003).

- Manutenção preventiva - ação que evita de alguma forma a deterioração ou interrupção futura de um sistema, equipamento ou parte deste. Consiste basicamente em inspeções periódicas e checagens de funções existentes de maneira a evitar falha no desempenho, bem como possibilitar a correção ainda em estágio inicial de processo de deterioração (ANTONIOLI, 2003).

- Relatórios realizados a partir de uma interface na web.

De uma forma geral, as ferramentas CAFM compõem um ambiente de organização, gerenciamento e comunicação de informações que constituem um banco de dados que organiza, armazena e distribui as informações sobre a edificação, seus sistemas, atividades e o espaço urbano. Porém destaca-se a importância da atualização constante da base de dados do sistema e a coerência das informações, para que possa funcionar de forma eficiente.

\section{CAMPUS UNIVERSITÁRIO - A ORIGEM}

Segundo Verger (1999) as primeiras universidades aparecem em Bolonha, em Paris, em Montpellier, em Oxford nos primeiros anos do século XIII. As instalações das universidades ocupavam geralmente o centro das cidades, como em Bolonha, Paris e Coimbra.

Não existiam prédios específicos para ministrar as aulas, eram espaços alugados e posteriormente passaram a acontecer nas hospedarias e espaços de ensino. A reunião de estudantes e mestres em um mesmo local exigiu naturalmente a instituição de normas de disciplina e conduta, estes espaços teriam que ser reorganizados para atender, de forma eficaz, à nova realidade (VERGER, 1990).

Afirma Verger, que no século $\mathrm{XV}$ as universidades almejavam possuir suas sedes. Na Europa, as universidades surgiram concomitantemente ao processo de urbanização, os espaços passaram por inúmeras modificações, implantados nos limites das cidades e os novos eram construídos próximos aos existentes e iam transformando-se em universidades, espaços independentes em meio à malha urbana, por exemplo, a Universidade de Oxford, conforme Figura 1.

\footnotetext{
${ }^{3}$ Constitui basicamente em um mecanismo computacional de pergunta e resposta, onde a pergunta feita pelo usuário, é consultada em uma base de dados, e retornada a resposta de um questionamento semelhante feito anteriormente (MATOS; FERNANDES; MORIRA, 2006).
} 
Figura 1 - Universidade de Oxford

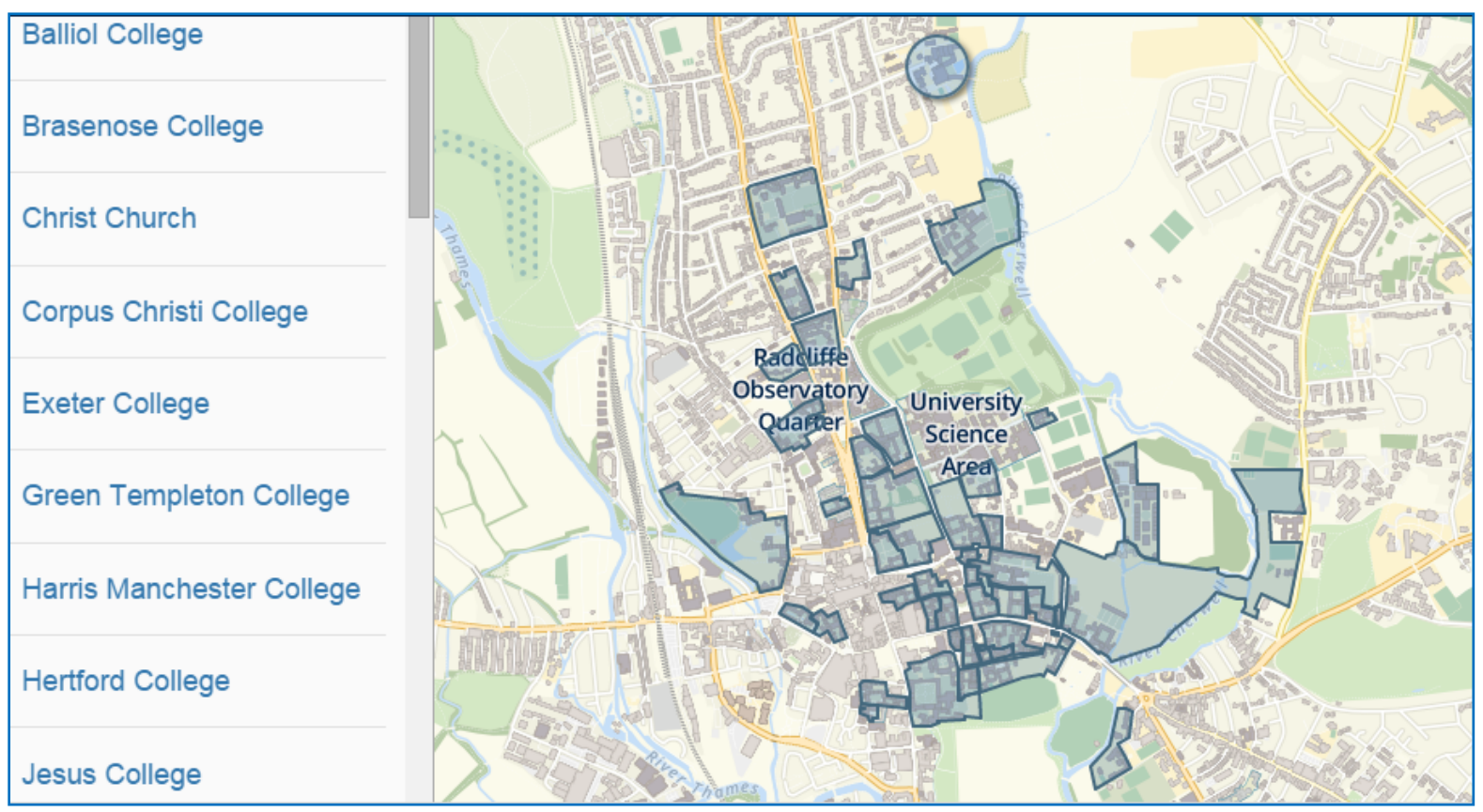

Fonte: http:// www.ox.ac.uk/visitors/maps-and-directions/searchable-map.

O território da universidade é definido por suas edificações e não por um sítio, ou melhor, uma área fechada e afastada da cidade. Ao redor dos seus edifícios a cidade flui livremente.

A educação superior americana é traçada desde o período colonial na concepção de colleges e universities, classificadas como cidades microscópicas. Não eram construídas apenas salas de aula, mas também dormitórios, refeitórios e espaços recreativos. $O$ trabalho do arquiteto não se resumia a projetar edifícios isolados, e sim o espaço para convívio da comunidade (TURNER, 1995). Ainda segundo o autor, o ideal americano era afastar as universidades das forças corruptoras das cidades. O college tornou-se um experimento de urbanismo, o campus era composto pela biblioteca e diversos edifícios, um espaço fechado caracterizando uma pequena cidade com equipamentos, serviços e recursos que uma cidade oferece, com leis e regras próprias.

Segundo Cunha (1983), o ensino superior no Brasil foi marcado por escolas isoladas, só após o final do Estado Novo é que as escolas americanas passaram a ser um modelo para as universidades brasileiras. O objetivo era transformar os campi em cidades universitárias, onde a pretensão era oferecer ensino, centros de pesquisa, acolher alunos e professores com todos os serviços que uma cidade oferece. Entretanto, isto não aconteceu desta forma, estes centros não são autossuficientes e dependem das cidades onde estão implantados.

\section{PLANEJAMENTO E GESTÃO DE CAMPI UNIVERSITÁRIOS}

Segundo Pinto e Buffa (2009) existe uma política histórica nos campi universitários das cidades brasileiras, de construir edifícios com o menor custo possível. Não se gasta tempo e dinheiro planejando e construindo edificações e instalações de qualidade, mas refazendo ou até demolindo as mesmas. Novas tecnologias significam maiores investimentos em 
infraestrutura e formação de mão-de-obra especializada, o que requer planejamento cuidadoso e mais recursos, de modo a minimizar maiores gastos com contratempos futuros.

Pinto e Buffa (2009) afirmam que o projeto de implantação das universidades brasileiras inspirado nas regras de zoneamento urbano como, por exemplo, na Universidade Federal do Rio de Janeiro (UFRJ), discutida e apresentada com base na Carta de Atenas ${ }^{4}$, previa uma organização por setores administrativos, unidades acadêmicas, alojamentos e serviços auxiliares, deveria ser um parque único, porém desde a sua concepção não previa serviços nem espaços que caracterizassem um núcleo urbano.

No Brasil é muito comum encontrar o histórico de universidades cujos projetos aspiravam reunir em um mesmo sítio, cursos existentes espalhados pela cidade. Entretanto, a exemplo da USP, que mesmo sendo uma universidade pública financiada pelo Estado mais rico da federação, cujo campus é extenso, bem equipado e administrado, ainda está longe de ser considerada uma cidade autônoma (PINTO; BUFFA, 2009).

\section{APLICAÇÕES DA TECNOLOGIA CAFM EM UNIVERSIDADES}

Quais as vantagens da utilização de um sistema que concentre as informações do campus visando a gestão da vida universitária? Conhecer melhor o território onde se trabalha, desenvolver projetos que sejam ajustados à realidade, saber quais os possíveis riscos e os desafios para serem superados. A grande vantagem é poder ter os dados mapeados e catalogados para serem apresentados aos gestores e à comunidade universitária, mas principalmente para o uso corrente e imediato nas mais diversas atividades. Conhecer a instituição para projetar o futuro é o primeiro passo para o cumprimento das suas finalidades.

Foram compilados alguns casos de aplicações de FM em universidades, a partir dos respectivos websites, com o objetivo de melhorar o processo que está sendo aplicado no estudo de caso do trabalho de pesquisa em andamento para o desenvolvimento de um protótipo de aplicação de facilities na Faculdade de Arquitetura da UFBA (FAUFBA).

\subsection{Centro Universitário FEEVALE (RS - BR)}

Neste centro foi adotado o facility management utilizando o software Archibus FM, e teve como objetivo manter atualizada a estrutura física da instituição e seus ativos, procurando estabelecer uma interface entre o banco de dados georreferenciado e o banco de dados alfanumérico. Foram necessários recursos humanos nas áreas de: gerenciamento de sistemas, programação, arquitetura, engenharia civil e administração (FURTADO, 2005).

\subsection{Xavier University (Ohio - USA)}

Este trabalho foi desenvolvido empregando modelos BIM e a aplicação de facilities através do software FM: Systems. O modelo BIM foi adaptado às necessidades da aplicação FM, foram removidos dados não relevantes no processo de gestão de facilities e acrescentados dados como, os códigos de classificação do espaço, geralmente acessados pelo pessoal de manutenção e chefes de departamentos.

\subsection{Indiana University (Indiana - USA)}

Benefícios da implantação de FM: redução de custos a partir de informações precisas para o

\footnotetext{
${ }^{4}$ Documento sobre o manifesto urbanístico resultante do IV Congresso Internacional de Arquitetura Moderna (CIAM), realizado em Atenas em 1933.
} 


\section{TIC2015}

desenvolvimento de novos projetos ou reformas e maior controle de alocação de espaços.

\subsection{Georgia Institute of Technology (Georgia - USA)}

O objetivo da aplicação de facilities foi desenvolver soluções para melhor aproveitamento dos espaços acadêmicos, melhor controle de ativos e administração dos serviços, tais como:

- Planejamento de recursos financeiros, orçamento e planejamento do desenvolvimento;

- Campus master planning - programação das futuras instalações;

- Planejamento de facilities - programação da manutenção e operação do campus;

- Gestão do patrimônio histórico - a partir do registro de documentos, fotos, plantas etc.;

- Sustentabilidade - energia renovável, eficiência energética, controle do meio ambiente;

- Programação de uso de espaços como as salas de aula e laboratórios;

- Programação de eventos;

- Arquivo digital de documentos, projetos, fotos, orçamentos etc.;

- Localização no campus de salas de aula, laboratórios, setores administrativos etc., a partir de um mapa, conforme Figura 2.

Figura 2 - Campus Georgia Tech: mapa 1

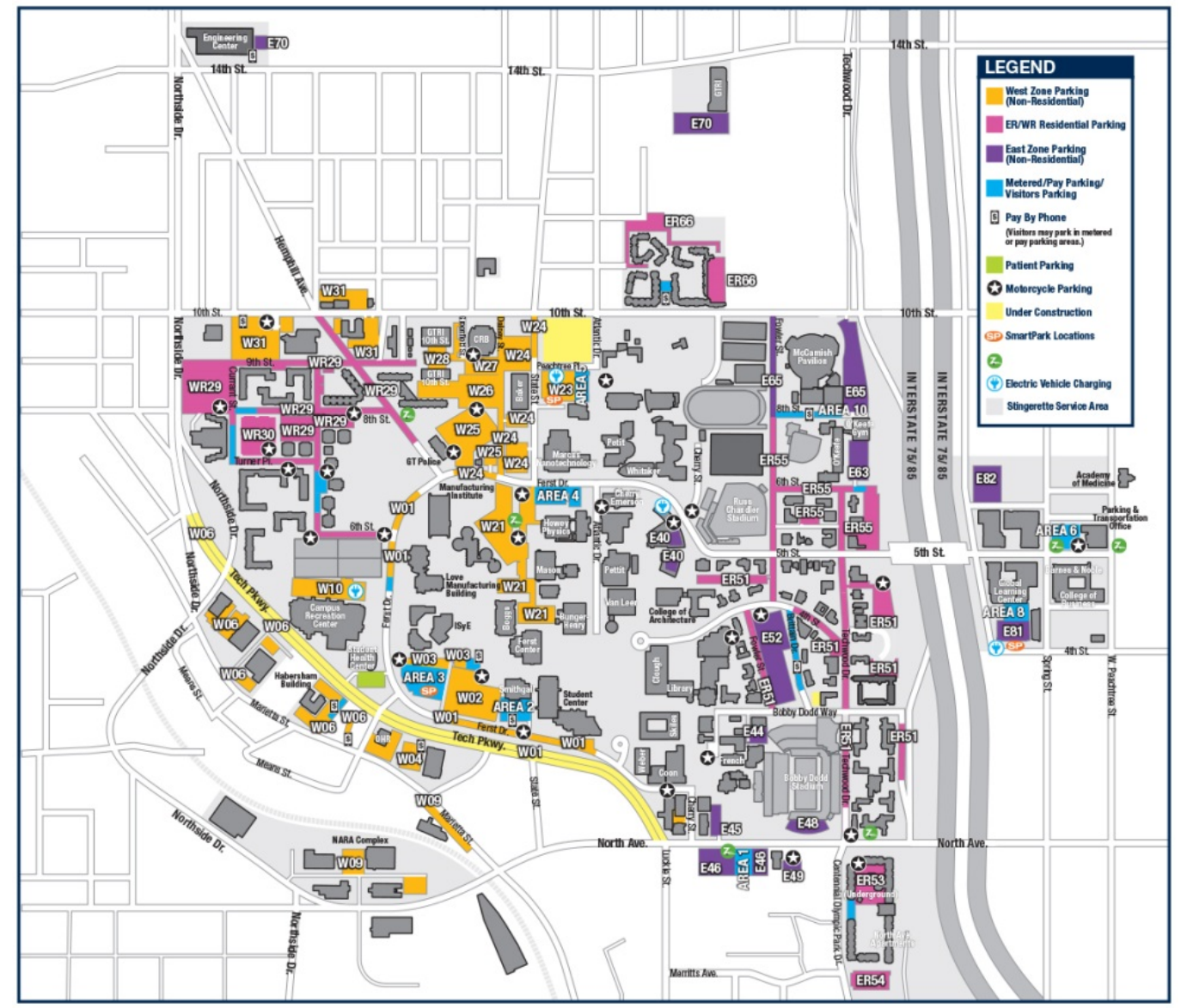

Fonte: http://pts.gatech.edu/Documents/ParkingZones2014.jpg

Mapa interativo com informações das edificações, serviços, área verde, estacionamento etc. 

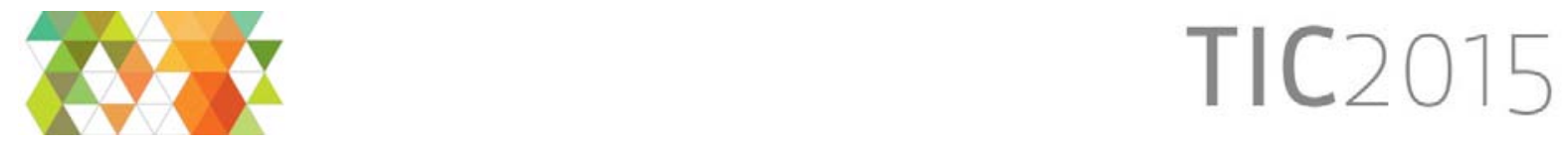

e outra legenda com transportes e vias de circulação (Figura 3).

Figura 3 - Campus Georgia Tech: mapa 2

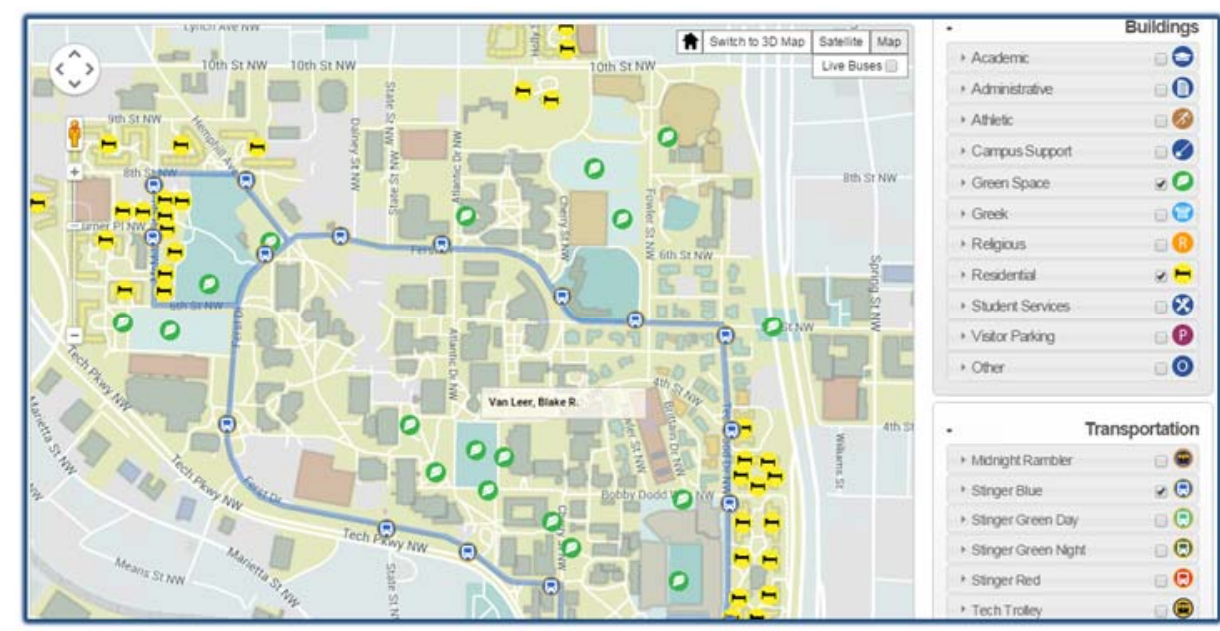

Fonte: http://maps.gatech.edu/d7/drupal/

- Plano Diretor do Campus da Georgia Tech (Figura 4).

Figura 4 - Mapa do plano diretor do campus da Georgia Tech

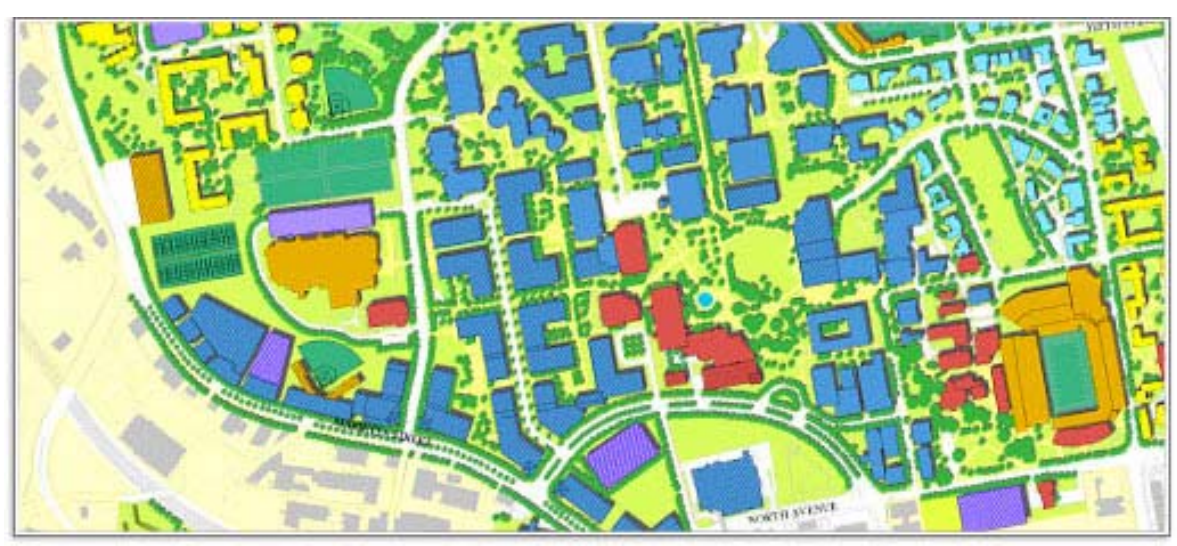

Fonte: http://www.space.gatech.edu/masterplan/

De acordo com a Georgia Tech, o plano diretor do campus foi elaborado em 1997 e é considerado um "documento vivo", dinâmico5. Pela dificuldade de prever todas as circunstâncias futuras foi necessário dota-lo de maior flexibilidade para se ajustar às mudanças que fossem surgindo. O master plan representa os parâmetros e orientações que anteveem o desenvolvimento futuro, baseado em influências internas e externas.

O processo de implantação de CAFM a que foi submetido o campus universitário sugere um refinamento ou alterações no Plano Diretor, passando por ajustes das diretrizes, dando um maior enfoque para as áreas de acessibilidade e sustentabilidade e disponibiliza-o para a comunidade acadêmica visualizar as atualizações constantes, assim como os planos anteriores.

\footnotetext{
${ }^{5}$ Elaborado a partir de uma detalhada análise das condições existentes e das futuras necessidades da universidade.
} 


\section{APLICAÇÃO DE CAFM NA FACULDADE DE ARQUITETURA - UFBA}

O mestrado que está em andamento tem como tema: Implantação de Facilities Management em instituições públicas de ensino superior, cujo objetivo principal é propor uma rotina de procedimentos do processo de implantação de FM na FAUFBA, e a implementação de um protótipo. Tal sistema busca aumentar a eficiência de uso da edificação, reduzir seus custos operacionais, facilitar a tomada de decisões administrativas e prolongar a vida útil do empreendimento e seus equipamentos.

Inicialmente foi realizada uma pesquisa bibliográfica, onde foi possível notar a escassez de material bibliográfico, principalmente nacional. Para a implementação do protótipo estão sendo desenvolvidos modelos BIM das edificações que compõem a FAUFBA. Para este fim foram levantados documentos cadastrais e projetos de futuras reformas, ajustando os desenhos ao as built. Os modelos estão sendo desenvolvidos no Revit, com uma atenção especial ao nível de detalhamento simplificado, com o objetivo de diminuir o tamanho dos arquivos e possíveis erros.

A FAUFBA é composta por seis edificações, foi modelado inicialmente o terreno e está em andamento a modelagem de cada edificação separadamente. Cada edifício será trabalhado no seu interior, identificando os ambientes através de códigos individuais, sem repetição para não haver erro na aplicação de CAFM.

Concomitantemente, estão sendo levantadas as informações que serão trabalhadas no protótipo utilizando o software Archibus FM, tais como código a ser adotado em cada espaço, área de cada ambiente, equipamentos associados aos espaços ou às pessoas, mobiliário e seus respectivos números de tombamento, especificações, termos de garantias, datas de garantias, manual de uso e manutenção. Neste processo serão levantadas as atividades que poderão ser atendidas pelo sistema CAFM, as pessoas associadas a estas atividades e os espaços alocados para atender a estas funções. Por se tratar de uma edificação pré-existente enfrentamos dificuldades em localizar alguns documentos, e a intenção será resgatar o máximo que for possível e criar um repositório para facilitar os acessos.

\section{CONSIDERAÇÕES FINAIS}

O avanço tecnológico impõe um ritmo acelerado às tomadas de decisões mediante informações constantemente atualizadas e a necessidade de expansão dos conhecimentos. A cada dia surgem mais desafios em áreas que envolvem múltiplas competências, facilmente encontrados no processo de gestão do campus universitário, onde seria de extrema relevância.

A pesquisa encontra-se em fase de implementação do estudo de caso e pretende contribuir com a rotina de procedimentos para a elaboração de um protótipo de aplicação CAFM em um complexo de edifícios que constituem as instalações da FAUFBA, onde espera-se concentrar informações relevantes na função de manter e operar a edificação, reforçando a cultura em prol dos cuidados e atenção para com a edificação e os usuários dentro do ciclo de vida específico de cada unidade.

\section{AGRADECIMENTOS}

Gostaria de agradecer à colaboração da FAUFBA - Direção e Administração, ao Prof. Fabiano Mikalauskas e alunos Pedro Mariano e Thiago Motta pela modelagem das edificações, a Denise Vaz da SUMAI por fornecer as plantas, ao Arq. Rogério Suzuki sempre solícito e à Archibus FM por fornecer a licença e o apoio ao desenvolvimento do trabalho. 


\section{REFERÊNCIAS}

Accruent. Disponível em: <http://www.accruent.com/resources/tags/saas>. Acesso em: 20 mar. 2015.

ANTONIOLI, P. E. Estudo crítico sobre subsídios conceituais para suporte do planejamento de sistemas de gerenciamento de facilidades em edificações produtivas. Dissertação (Mestrado) Escola Politécnica, Universidade de São Paulo, São Paulo, 2003. 241 p.

Archibus FM. Disponível em: <http://www.archibus.com/success-story-by-application/section/ Success\%20Stories/menuid/60>. Acesso em: 22 mar. 2015.

CÂMARA, G.; MEDEIROS, J. Geoprocessamento para projetos ambientais. 2. ed. São José dos Campos: INPE, 1998. 194 p.

CUNHA, L. A. A Universidade Crítica: o ensino superior na República populista. Rio de Janeiro: Francisco Alves, 1983.

EuroFM (European Facility Management Network). Disponível em: <http://www.eurofm.org/aboutus/what-is-fm/>. Acesso em: 27 out. 2013.

FERREIRA, F. P. Gestão de facilities: estudo exploratório da prática em empresas instaladas na região metropolitana de Porto Alegre. 2005. 152 f. Dissertação (Mestrado em Construção Civil) Universidade Federal do Rio Grande do Sul, Porto Alegre, 2005. Disponível em: http://hdl.handle.net/10183/6527. Acesso em: 25 jan. 2012.

FM Systems. Disponível em: <http://www.fmsystems.com/>. Acesso em: 20 mar. 2015.

FURTADO, M. F. et al. Gerenciamento de facilidades na Unidade II do Centro FEEVALE, Novo Hamburgo, RS. São Paulo: Ed. Exacta, v. 3, p. 95-102, 2005.

Georgia Institute of Technology. Disponível em: <http://pts.gatech.edu/Pages/default.aspx>. Acesso em: 20 mar. 2015.

IFMA - Associação Internacional Facility Management. Disponível em: <http://www.ifma.org/ about/what-is-facility-management>. Acesso em: 02 ago. 2015.

Indiana University (IU). Disponível em: <http://www.iub.edu/>. Acesso em: 20 mar. 2015.

Maintenance Management for Schools and Universities. Disponível em: <http://www.

maintenanceconnection.com/website/industries/facility-management/school-universities/>. Acesso em: 20 mar. 2015.

MATOS, F.; FERNANDES, A. M. R.; MORIRA, B. G. Sistema de Help Desk Utilizando RBC - Um estudo de caso sobre o software legal. In: Congresso Sul Brasileiro de Computação, Itajaí, SC, 2006. Disponível em: <http://periodicos.unesc.net/index.php/sulcomp/article/view/1000/937>. Acesso em: 7 maio 2015.

PINTO, G. A.; BUFFA, E. Arquitetura e educação: campus universitários brasileiros. São Carlos: EESC-USP / UFSCar, 2009. 151 p.

QUINELLO, R.; NICOLETTI, J. R. Gestão de Facilidades. São Paulo: Novatec, 2006. 264 p.

Total Facilities Management (TFM). Disponível em: <http://www.ocs.co.uk/Total-FacilitiesManagement>. Acesso em: 3 nov. 2013.

TURNER, P. V. Campus: an american planing tradition. MIT Press, 1995.

VERGER, J. As Universidades na Idade Média. São Paulo: UNESP, 1990.

Homens e Saber na Idade Média. Tradução Carlota Boto. Bauru, SP: EDUSC, 284 p. 1999.

WATSON, James; WATSON, Russ. Computer-Aided Facilities Management (CAFM), 2013.

Disponível em:< https://www.wbdg.org/om/cafm.php>. Acesso em: 03 ago. 2015. 
WEISE, A. D. et al. Os Conceitos do Custo de Ciclo de Vida de Imóveis Aplicado no Facility Management. In: Simpósio de Excelência em Gestão e Tecnologia, 6., 2009, Resende. Anais eletrônicos... Rio de Janeiro: AEDB, 2009. Disponível em: <http://www.aedb.br/seget/arquivos/ artigos09/408_artigofinal_1.pdf>. Acesso em: 8 maio 2015. 